\title{
Comparison of wavelength propagation for free space optical communications
}

\begin{abstract}
This paper presents the effect of atmospheric attenuation during severe hazy days for Free Space Optical Communications. The usage of Free Space Optical Communications is still rare in Malaysia due to environment factor. The FSO technology is also known as unguided beam or óptical wirelessô or infrared broadband. This study offers quick preliminary investigation on possible FSO performance based on wavelength selection before final commissioning and installation at site. Thus, FSO installers could make quick judgment before giving recommendation of a suitable wavelength to the customers. Preliminary evaluation of system performance of system performance is done by using local weather data obtained from metrological department. Current study among designers and FSO users show that $1550 \mathrm{~nm}$ light produce less atmospheric attenuation in the transmission under all weather conditions. In this study, a suitable wavelength for FSO system is found for a particular site in low visibility. The best wavelength selection would result in optimized quality of FSO transmission in hazy conditions.
\end{abstract}

Keyword: FSO; Free space optical; Mie scattering; Scattering coefficient; Atmospheric attenuation 